\title{
RESEARCH DATA TRANSPARENCY AND OPENNESS FOR THE PROCEEDINGS OF THE NOVA SCOTIAN INSTITUTE OF SCIENCE
}

\author{
ANDREW SHERIN ${ }^{1 *}$, MARY KENNEDY ${ }^{2}$, \\ AND ALEXI BACCARDAX WESTCOTT ${ }^{1}$ \\ ${ }^{1}$ Atlantic Coastal Zone Information Steering Committee Secretariat, \\ Dalhousie University, Halifax, NS \\ ${ }^{2}$ Ocean Biogeographic Information System (OBIS Canada)
}

This paper addresses the issue of the transparency of the original research data and accessibility to it (i.e. openness) for each published paper in the Proceedings of the Nova Scotian Institute of Science (PNSIS).

In 2004, member nations of the Organization for Economic Cooperation and Development (OECD), including Canada, signed a declaration on Access to Research Data from Public Funding. In that declaration, they recognized four principles: (1) An optimum international exchange of data, information and knowledge contributes decisively to the advancement of scientific research and innovation; (2) Open access to, and unrestricted use of, data promotes scientific progress and facilitates the training of researchers subject to appropriate constraints for national security, the protection of privacy of citizens and the protection of intellectual property rights and trade secrets; (3) Open access will maximise the value derived from public investments in data collection efforts; and (4) The substantial increase in computing capacity enables vast quantities of digital research data from public funding to be put to use for multiple research purposes by many research institutes of the global science system, thereby substantially increasing the scope and scale of research. The declaration also recognized the substantial benefits that science, the economy and society at large could gain from the opportunities that expanded use of digital data resources have to offer, and the risk that undue restrictions on access to and use of research data from public funding could diminish the quality and efficiency of scientific research and innovation. 
The Atlantic Coastal Zone Information Steering Committee's (ACZISC) project, "Atlantic Canada's Biological Data for Ecosystem Planning and Decision-making: Opening Access and Increasing Reuse", is working with partners to improve the public accessibility to marine biogeographic data in Atlantic Canada. This is accomplished through publishing datasets with the Canadian node of the Ocean Biogeographic Information System (OBIS Canada).

The data of interest for the project are biological species occurrence (scientific name and geographic location) in a standard format and with standard documentation. The OBIS, created by the international Census of Marine Life program (2000-2010), is now part of the Intergovernmental Oceanographic Commission (IOC) of UNESCO, under its International Oceanographic Data and Information Exchange (IODE) programme.

The ACZISC project is funded by Environment Canada's Atlantic Ecosystem Initiative. In 2014-2015, four partner organizations contributed data: the Nova Scotia Museum of Natural History in Halifax, Nova Scotia; the Bras d'Or Institute at Cape Breton University, Sydney, Nova Scotia; the Atlantic Reference Centre, Huntsman Marine Science Centre, St. Andrews, New Brunswick; and the Southern Gulf of St. Lawrence Coalition on Sustainability, Charlottetown, Prince Edward Island. An objective of the project is to publish a State of Biological Data Accessibility Report and to establish an ongoing partnership of organizations in the Atlantic Region interested in improving accessibility to the biological data that they hold.

In Canada, Research Data Canada (RDC) (http://www.rdc-drc. ca), is a forum in which multiple stakeholders, including research funding agencies, come together to build research data stewardship. RDC focusses "on ensuring research data is accessible, interoperable and appropriately preserved so that it may be reused by Canadians."

Depositing data from scientific articles published in journals in a publically accessible repository is now a common requirement of publishers. The Centre for Open Science (https://cos.io/ ) documents 538 journals that are signatories to their Transparency and Openness Promotion (TOP) Guidelines (COS nd, Nosek et al. 2015). The TOP standard for data transparency requires authors submitting articles to journals to post the data to a trusted repository to meet the stringent levels 2 and 3 of the standard. OBIS would qualify as a "trusted repository" for marine taxa. The Global Biodiversity Information Facility (GBIF) performs a similar function for terrestrial and fresh water taxa. 
Datasets deposited with OBIS Canada can be freely accessed by the general public, OBIS, GBIF and other initiatives.

Only some data supporting the PNSIS articles would be considered biogeographic in nature. Repositories other than OBIS and GBIF would need to be identified for other data types. That notwithstanding, the transparency of and accessibility to the original research data of each published paper should be a consideration for the NSIS and the PNSIS Editorial Board. The authors encourage readers to send comments to the President of NSIS and the Editor on the possible formal adoption of the research data accessibility principles of the OECD declaration and the Centre for Open Science TOP Guidelines (described briefly above) for the PNSIS.

\section{REFERENCES}

Center for Open Science. (nd). Transparency and Openness Promotion (TOP) Guidelines https://cos.io/top/.

Nosek, B.A., Alter, G., \& Banks, G.C., et al. (2015). Promoting an open research culture. Author guidelines for journals could help to promote transparency, openness, and reproducibility. Science 348 (6242):14221425. 26 June, 2015.

OECD. (2004). OECD Declaration on Access to Research Data from Public Funding. 30 January 2004. http://acts.oecd.org/Instruments/Show InstrumentView.aspx?InstrumentID=157. 